\title{
Nach tiefer Beinvenenthrombose: drei Monate Antikoagulation genügen
}

- Die gepoolte Analyse von sieben großen randomisierten Studien ergibt, dass eine dreimonatige Antikoagulation für distale tiefe Beinvenenthrombosen (TVT) zur Rezidivprophylaxe ausreichend ist. Proximale Thrombosen ohne erkennbare Risikofaktoren und Thrombosen mit Lungenembolie haben dagegen ein hohes Rezidivrisiko, wann immer die Antikoagulation gestoppt wird.

Über die optimale Dauer der Antikoagulation nach TVT gibt es nach wie vor Diskussionen. Die klinischen Situationen und Risikokonstellationen sind sehr heterogen und erfordern daher in Studien große Patientenzahlen, um zu validen Aussagen zu kommen. Nun hat man sieben randomisierte Studien in einer gepoolten Analyse ausgewertet. Auf diese Weise konnten die Daten von 2925 Männern und Frauen ohne maligne Erkrankung mit der ersten Episode einer TVT und verschiedenen antikoagulatorischen Therapiemodalitäten ausgewertet werden.

Die Rezidivneigung war bei isolierten distalen TVT nur halb so hoch wie bei proximalen. Patienten mit proximalen TVT und solche mit Lungenembolie hatten ein gleich hohes Risiko. Lagen temporäre Gründe für das Auftreten der Thrombose vor, zum Beispiel eine Immobilisierung durch Gips, so betrug das Rezidivrisiko im Vergleich zur spontan aufgetretenen TVT ebenfalls nur 50\%.

Das Optimum der Dauer der Antikoagulation lag bei drei Monaten. Kürzere Zeitdauern gingen mit einem erhöhten Rezidivrisiko einher, längere erbrachten bezüglich des Rezidivrisikos keinen Vorteil, erhöhten aber das Blutungsrisiko.

Die spontan aufgetretene proximale TVT und Patienten mit Lungenembolie haben immer ein hohes Rezidivrisiko, wann immer man die Antikoagulation beendet. Daher empfiehlt es sich, bei dieser Patientengruppe eine lebenslange Antikoagulation in Erwägung zu ziehen. Erhöhte D-Dimer-Konzentrationen während oder nach einer Antikoagulationstherapie scheinen ein wertvoller Prädiktor für ein Wiederauftreten einer TVT zu sein, zumindest bei den Pati- enten mit spontan aufgetretener Thrombose ohne einschlägige Risikofaktoren.

\section{Kommentar}

Die Entscheidung über die Zeitdauer der Antikoagulation ist komplex und wird von zahlreichen Faktoren beeinflusst, die nicht immer scharf definiert, vielleicht auch nicht definierbar sind. Daher bietet die Untersuchung zwar viele gute Anhaltspunkte für das optimale Vorgehen, es dürften aber immer noch Situationen auftreten, bei denen das Vorgehen nicht durch klare Evidenzen festgelegt ist. Das Ermessen des Arztes, die Wünsche des Patienten und das Prinzip Hoffnung dürften bei der Therapie der tiefen Beinvenenthrombose nicht vollständig abzulösen sein.

H. S. FÜESSL

\footnotetext{
- F. Boutitie et al.

(Korresp.: C Kearon, Hamilton Health Scienes, Henderson Division, 711 Concession Street, Hamilton, ON Canada L8V 1C3; kearonc@ mcmaster.ca): Influence of preceding length of anticoagulant treatment and initial presentation of venous thromboembolism on risk of recurrence after stopping treatment: analysis of individual participants' data from seven trials. Brit. Med. J. 342 (2011) d3036
}

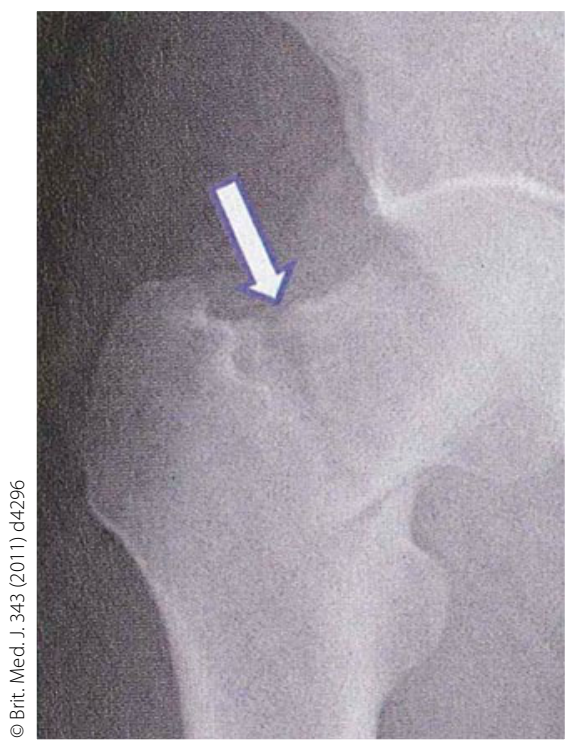

Stressfraktur des Schenkelhalses bei einem Marathonläufer.

\section{Durchbruch beim Marathon}

- Nach der Teilnahme am London Marathon 2011 suchte ein 54-jähriger Läufer wegen starker Schmerzen im Bereich der rechten Hüfte die Nothilfe auf. Er hatte bereits während des Trainings in den zwei Monaten vor dem Rennen immer wieder einmal Beschwerden gehabt. Bei etwa $36 \mathrm{~km}$ hatten die Schmerzen während des Laufes massiv zugenommen und er rettete sich mehr oder weniger humpelnd über die Ziellinie.

Das Röntgenbild zeigte eine durchgehende bikortikale Stressfraktur des Schenkelhalses, die mit einer dynamischen Hüftkopfschraube versorgt wurde. Stressfrakturen des Schenkel- halses sind bei Athleten nicht ungewöhnlich und können, wenn sie frühzeitig entdeckt werden und nur eine Corticalis betreffen, konservativ durch Entlastung behandelt werden. Vernachlässigt man diese Verletzung, so ist eine operative Behandlung unvermeidbar. Wahrscheinlich wäre der Läufer gut beraten gewesen, sich aufgrund der Schmerzen bereits während des Trainings vor dem Marathon untersuchen zu lassen.

H. S. FÜESSL

\footnotetext{
- S Newman et al.

(Dept. of Trauma and Orthopaedics, St Mary's Hospital, Praed Street, London W2 1NY) Brit. Med. J. 343 (2011) d4296
} 